\title{
Development of a Mechanical Creative Combination Based on 3D Printing
}

\author{
Xinwen Li, Yingjie Tang* \\ College of Nuclear Technology and Automation Engineering, Chengdu University of \\ Technology, Chengdu 610059, China \\ *Corresponding author e-mail: tngyjie@163.com
}

\begin{abstract}
D printing technology, as a material manufacturing technology, is widely used in film, education, mechanical design, industrial design, medical, aviation, mechanical automation and other fields, in the process of education and mechanical design, 3D printing technology show extraordinary ability. Through this $3 D$ printing-based manufacturing method, a cam-driven handling manipulator suitable for teaching is developed and designed. The reciprocating motion of the manipulator is carried out by the joint action of the two cams, and the subsequent connecting rod mechanism makes the manipulator With the steering function, using SolidWorks simulation analysis, the impact of smaller institutions, better stability, to meet the design requirements, in order to adapt to teaching use, part of the box using plexiglass material, making the entire design can be appreciated.
\end{abstract}

KEYWORDS: 3D printing, mechanical creative combination, cam-driven manipulator, linkage mechani

\section{Introduction}

The development of mechanical creative combination refers to a practice-based development process in which the designer makes full use of creativity, innovates the existing mechanical structure, and designs a mechanical combination structure with creativity, feasibility and enjoyability. 3D printing technology, the so-called additive manufacturing (AM) [1], is a three-dimensional model file based on the use of high-energy beam source and other special methods, resin, metal powder, ABS, PLA, etc [2]. 3D printing The material is layered and bonded layer by layer, superimposed and formed into a single body. Unlike traditional manufacturing processes based on manufacturing reduction (such as turning, milling, planing, grinding, etc.), 3D printing technology is a manufacturing process that stacks materials layer by layer.

Combined with the application of 3D printing in the field of education at home and abroad, it is known that cultivating the innovative ability and scientific literacy

Published by Francis Academic Press, UK 
of the educated is the essence of 3D printing technology applied to education [3]. The mechanical creative combination can be used as a teaching tool to enable students to have a deeper understanding of knowledge in physical objects, or as a teaching purpose, allowing students to develop their own mechanical creative combination based on existing knowledge. Therefore, 3D printing technology can be applied to the classroom to teach students how to design and print a mechanical creative combination, or to apply 3D printing technology to cultivate students' creative thinking [4] [5], so that students can independently develop mechanical creative combinations.

In view of the importance of the above-mentioned mechanical creative combination in teaching equipment, this paper proposes a 3D printing technology to allow students to use teaching aids to learn and perform some imitation production. In this process, a cam-driven robot is used as an example. A deep understanding of the knowledge of mechanical principles, so as to get a better teaching effect.

\section{The origin of teaching aid development}

The manipulator mainly consists of an actuator, a drive mechanism and a control mechanism. The law of motion is the characteristic of the cam mechanism during the movement [6]. This design selects the design-based manufacturing technology of $3 \mathrm{D}$ printing. From the perspective of teaching, according to the characteristics of the cam-driven manipulator, the appropriate motion law is selected to complete the overall design of the cam-driven manipulator. The main creative point of this design is to control the entire stroke movement of the manipulator through a master axis, and the movement process does not interfere, and a certain steering placement function can be realized. The specific model is shown in Figure 1.

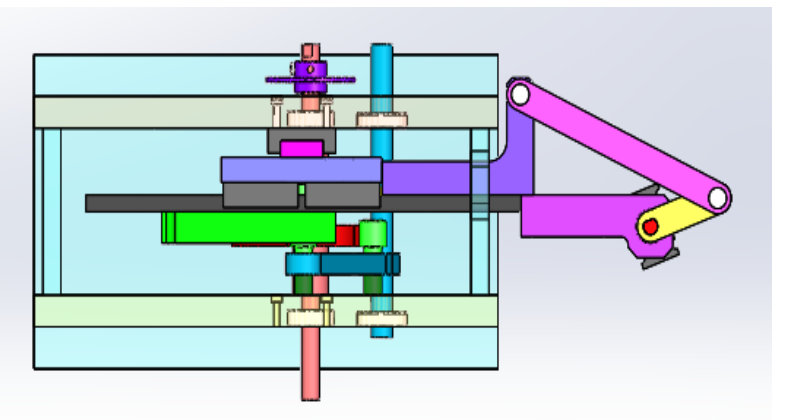

Figure. 1 Cam-driven manipulator

\section{Design requirements and solutions}

This robot is mainly used for teaching purposes. The main purpose is to enable students to deeply understand and study the cam mechanism [7]. Therefore, it pays

Published by Francis Academic Press, UK 
more attention to the influence of cam contour on the trajectory of the manipulator. For this design purpose, a horizontal stroke of $80 \mathrm{~mm}$ and a vertical stroke of $15 \mathrm{~mm}$ are required. And in order to highlight the innovation of the robot, a function of steering placement is required.

According to the above design requirements and combined with the knowledge of mechanical principles, it is known that the in-plane movement should be designed first, and then the steering function completed during the translation process should be designed, and the specific planar motion cycle trajectory is obtained as shown in Figure 2.

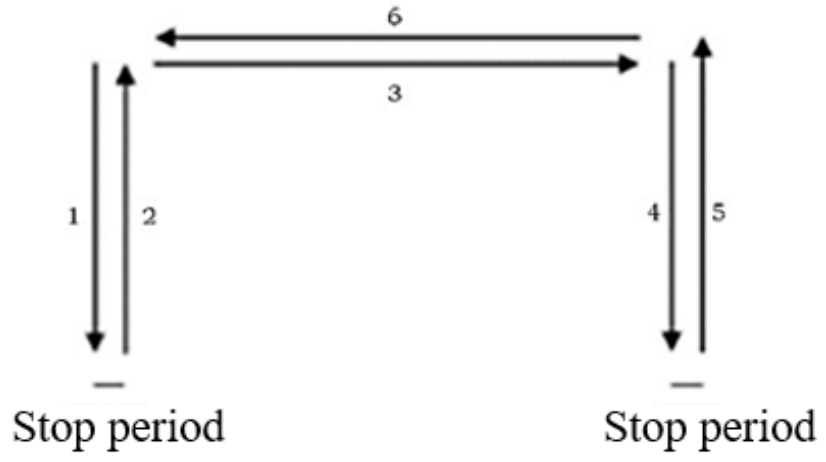

Figure. 2 Motion cycle diagram

It can be seen from analysis of Fig. 2 that in the process of 1, 2, 4, and 5, the robot will move up and down, and the maximum distance of lifting and lowering is the vertical stroke of the robot. 3, 6 The manipulator will move in translation during the process, and the maximum distance of translation is the horizontal stroke of the manipulator. It can be seen that the motion path of the cam-driven manipulator is divided into two motion processes, horizontal and vertical. Two cams are required to control the horizontal and vertical motion respectively. This design hopes to control the two cams with only one driving device. And the control motion of the two cams does not interfere, in this case, the student can conduct research and analysis on the two cams at the same time, in order to have a deeper understanding of the knowledge of the cam mechanism. Because this design hopes to be used as a teaching aid in teaching, it needs to meet the design requirements, reasonable structure and certain inspiration of teaching aids. The following two solutions can be obtained through analysis.

Scheme 1: As shown in Fig. 3, two disc cams are used, respectively, so that the follower drives the manipulator to perform translation and vertical movement, and the two motion processes are combined to enable the robot to complete the predetermined trajectory.

Published by Francis Academic Press, UK 
Scheme 2: As shown in Fig. 4, two arc cams are used. The driven disc has two rollers whose axes are radially distributed along the turntable. The contour surface pushes the swing of the follower to realize the required movement track of the robot.

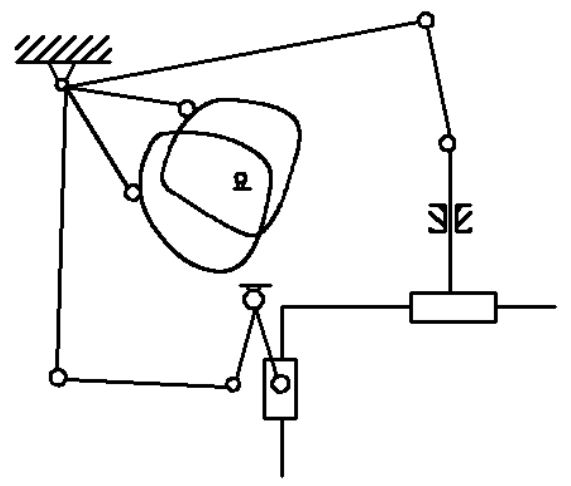

Figure. 3 Schematic diagram of the scheme 1

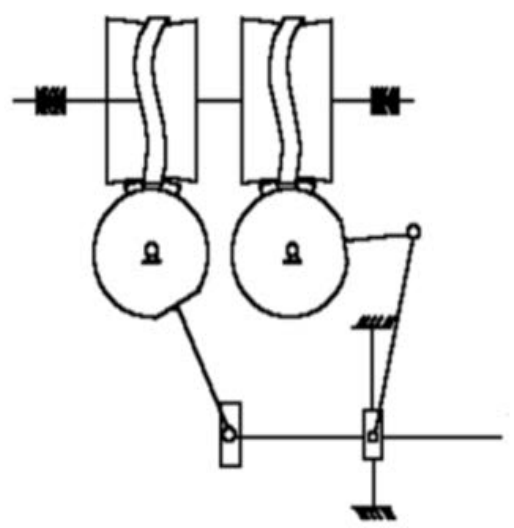

Figure. 4 Schematic diagram of scheme 2

In summary, the structure of the scheme 1 is more complicated, but the cam design is simpler and the motion process is convenient to analyze. The second scheme is simple in structure, but the space cam is required as the driving mechanism, and the cam design is complicated. Since the design is for the purpose of teaching mechanical principles, and more disc-shaped cams are used as learning targets in the mechanical principle, a wider planar disc cam is used as the driving mechanism, that is, the first scheme is adopted. 


\section{Cam driven robot design}

\subsection{Cam design}

The cam mechanism works by the contour curve of the outer edge of the cam [8], that is, the cam contour determines whether the cam mechanism can move according to a predetermined trajectory, which is the key to the design of the entire cam mechanism.

The double cam linkage mechanism can utilize the angle of repose of the cam to change the continuous rotation into intermittent motion [9]. By the cooperation between the driving shaft and the upper key groove of the cam, the two cams are mounted at a fixed angle, so that the rest of the two cams are staggered, and the expected motion trajectory is achieved without mutual interference of motion [10]. It saves the space of the whole organization in the case of embodying creativity. Thus, the cam work sequence table 1 can be obtained by analyzing the motion cycle diagram.

Table 1 Cam work sequence table

\begin{tabular}{|c|c|c|c|}
\hline 1 & $\begin{array}{l}\text { The lifting cam lowers the manipulator from } \\
\text { A to } \mathrm{B} \text {, and the translation cam is at the near } \\
\text { angle of repose }\end{array}$ & $\begin{array}{l}\text { Ready to drop } \\
\text { and grab }\end{array}$ & $\begin{array}{l}\text { Drive shaft } \\
\text { rotation } \varphi_{1}\end{array}$ \\
\hline 2 & The robot stops at B & Grab items & $\begin{array}{l}\text { Drive shaft } \\
\text { rotation } \varphi_{2}\end{array}$ \\
\hline 3 & $\begin{array}{l}\text { The lifting cam raises the manipulator from B } \\
\text { to A and the translation cam is near the angle } \\
\text { of repose }\end{array}$ & Lifting items & $\begin{array}{l}\text { Drive shaft } \\
\text { rotation } \varphi_{3}\end{array}$ \\
\hline 4 & $\begin{array}{l}\text { The lifting cam enters the far angle of repose, } \\
\text { and the translation cam shifts the manipulator } \\
\text { from A to C }\end{array}$ & Moving goods & $\begin{array}{l}\text { Drive shaft } \\
\text { rotation } \varphi_{4}\end{array}$ \\
\hline 5 & $\begin{array}{c}\text { The lifting cam lowers the manipulator from } \\
\mathrm{C} \text { to } \mathrm{D} \text {, and the translation cam is at a far } \\
\text { angle of repose. }\end{array}$ & Ready to drop & $\begin{array}{l}\text { Drive shaft } \\
\text { rotation } \varphi_{5}\end{array}$ \\
\hline 6 & The robot stops at D & Place goods & $\begin{array}{l}\text { Drive shaft } \\
\text { rotation } \varphi_{6}\end{array}$ \\
\hline 7 & $\begin{array}{l}\text { The lifting cam raises the manipulator from } \mathrm{D} \\
\text { to } \mathrm{C} \text {, and the translation cam is at a far angle } \\
\text { of repose. }\end{array}$ & Dead return & $\begin{array}{l}\text { Drive shaft } \\
\text { rotation } \varphi_{7}\end{array}$ \\
\hline 8 & $\begin{array}{l}\text { The lifting cam enters the far angle of repose, } \\
\text { and the translation cam shifts the manipulator } \\
\text { from } \mathrm{C} \text { to A }\end{array}$ & Dead return & $\begin{array}{l}\text { Drive shaft } \\
\text { rotation } \varphi_{8}\end{array}$ \\
\hline
\end{tabular}

1-8 is a duty cycle, and $\varphi_{1}+\varphi_{2}+\varphi_{3}+\varphi_{4}+\varphi_{5}+\varphi_{6}+\varphi_{7}+\varphi_{8}=360^{\circ}$ 
As can be seen from Table 1, the lifting cam has four angles of repose, while the translation cam has only two angles of repose. For the design of a drive shaft, the two cams have a common angle of repose, that is, the two cams have a common angle of repose of $25^{\circ}$ during the rest period, so the master shaft should complete the remaining six in the remaining $310^{\circ}$. Work process. If the time for completing one stroke in the lifting and lowering directions is $t_{h x}, t_{h z}$, the corresponding cam stroke angles are $\theta_{\mathrm{hx}}, \theta_{\mathrm{hz}}$, assuming that the two directions take the same motion law and the accelerations are equal.

By formula:

$$
\left\{\begin{array}{c}
\mathrm{a}_{\mathrm{mx}}=\mathrm{a}_{\mathrm{mz}} \\
\mathrm{a}_{\mathrm{mx}}=\mathrm{h}_{\mathrm{x}} * \frac{\mathrm{A}}{\mathrm{t}_{\mathrm{hx}}{ }^{2}} \\
\mathrm{a}_{\mathrm{mz}}=\mathrm{h}_{\mathrm{z}} * \frac{\mathrm{A}}{\mathrm{t}_{\mathrm{hz}}{ }^{2}} \\
\mathrm{~W}_{\mathrm{d}}=\frac{\theta_{\mathrm{hx}}}{\mathrm{t}_{\mathrm{hx}}}=\frac{\theta_{\mathrm{hz}}}{t_{\mathrm{hz}}}
\end{array}\right.
$$

Available:

$$
\left\{\begin{array}{c}
\mathrm{h}_{\mathrm{x}}: \mathrm{h}_{\mathrm{z}}=\mathrm{t}_{\mathrm{hx}}{ }^{2}: \mathrm{t}_{\mathrm{hz}}{ }^{2} \\
\theta_{\mathrm{hx}}: \theta_{\mathrm{hz}}=\sqrt{\mathrm{h}_{\mathrm{x}}}: \sqrt{\mathrm{h}_{\mathrm{x}}}=1: 0.43
\end{array}\right.
$$

Solved:

$$
\left\{\begin{array}{l}
\theta_{\mathrm{hx}} \approx 83.3^{\circ} \\
\theta_{\mathrm{hz}} \approx 35.8^{\circ} \\
\theta_{\mathrm{hx}}=80^{\circ} \\
\theta_{\mathrm{hz}}=40^{\circ}
\end{array}\right.
$$

Since the design uses a dome follower to reduce the friction between the follower and the cam, and improve the reliability of the mechanism, there may be a certain error in the contact between the cam and the follower, in order to highlight the near angle of the cam, The stroke angle of the cam adopts $\theta_{\mathrm{hx}}=70^{\circ}, \theta_{\mathrm{hz}}=$ $30^{\circ}$, and the improved cycle process diagram is as shown in Fig. 5.

Published by Francis Academic Press, UK 


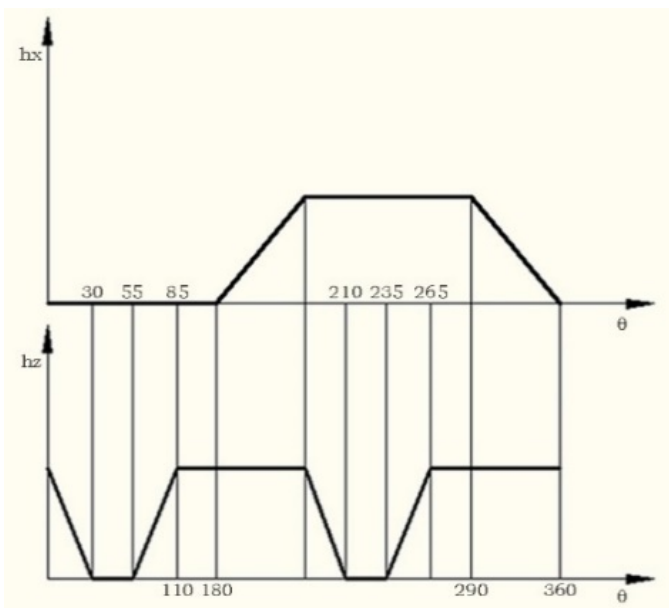

Figure. 5 Improve the cycle process

\subsection{Main parameters of the cam}

Because the design is used for teaching, the students can observe the motion law of the cam mechanism from the details, and use the rotating drive shaft to drive, so the detailed data such as the speed and angular velocity of the cam are not analyzed. According to the design of the above part, the contour of the cam can be designed. For the specific parameters, refer to Table 2.

Table 2 Main parameters of the cam

\begin{tabular}{|c|c|c|}
\hline Cam type & Lifting cam & Translation cam \\
\hline Size type & $15 \mathrm{~mm}$ & $80 \mathrm{~mm}$ \\
\hline stroke & $40^{\circ}$ & $40^{\circ}$ \\
\hline pressure angle & $30^{\circ}$ & $70^{\circ}$ \\
\hline Push motion angle & $30^{\circ}$ & $70^{\circ}$ \\
\hline Return movement angle & $25^{\circ}$ & $110^{\circ}$ \\
\hline Near angle of repose & $95^{\circ}$ & $110^{\circ}$ \\
\hline Far end angle & $30 \mathrm{~mm}$ & $25 \mathrm{~mm}$ \\
\hline Base circle radius & $45 \mathrm{~mm}$ & $45 \mathrm{~mm}$ \\
\hline Maximum diameter & &
\end{tabular}

\subsection{Selection of the motion law of the follower}

The law of constant velocity motion has a sudden change in the velocity of the follower at the start and end positions of the motion, so the inertial force is large and a rigid impact is generated. The quadratic polynomial motion law has a continuous 
speed curve, and the acceleration will produce sudden changes and flexible impacts, which are generally used in medium speed applications. When the rule of motion of the trigonometric function selects the law of cycloidal motion and corrects the law of sinusoidal motion [11], its jerk curve will abruptly change, while the displacement, velocity and acceleration curves will have sharp points, the convergence is not smooth, and the harmonic motion law The displacement, velocity, acceleration and jerk curves are continuous, and under the same design requirements, the peak value is relatively small, which is suitable for the motion law of lift-stopdown-stop. Therefore, the cam in the cam mechanism of the design adopts a simple harmonic motion law.

\subsection{Design of the remaining related parts}

(1) Follower. Assuming that the follower fixing point is c, the contact point of the follower and the cam is b, and the connection point of the follower and the slider portion is a. Since the difference between the maximum diameter and the base circle radius of the translation cam is $20 \mathrm{~mm}$, and the translational stroke is $80 \mathrm{~mm}$, the ratio of the total length ac of the bc and the follower is $1: 4$, and the bc distance is set to $45 \mathrm{~mm}$, the follower is set. The total length ac is $180 \mathrm{~mm}$. Considering the overall spatial distribution, it can be properly bent when the ac is $180 \mathrm{~mm}$, so that ab and bc are at a certain angle, which can save space and save materials. The specific design is shown in Fig. 6.

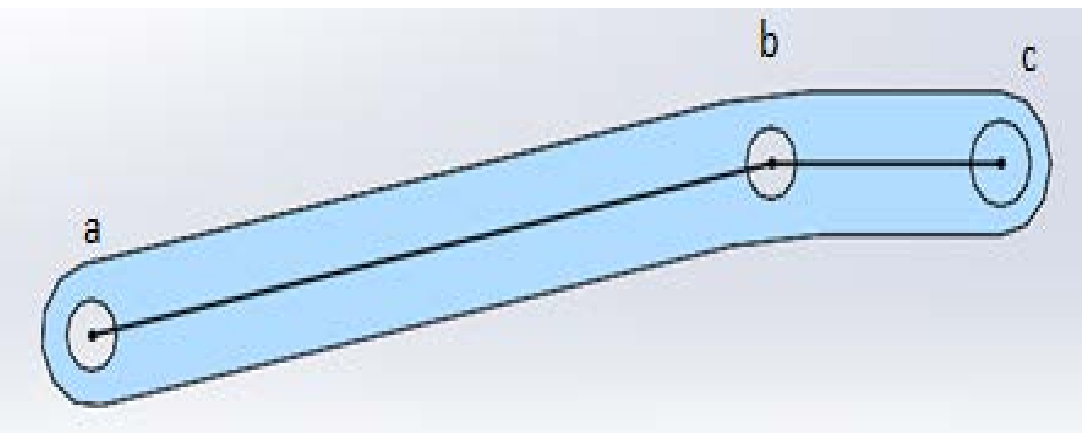

Figure. 6 Swing follower

(2) The drive shaft. Generally, the cam and the drive shaft need to be connected by a key and a keyway. The keyway must be completed by milling during machining, and then the key is added to transmit torque so that the drive shaft drives the cam to rotate. The 3D printing can directly print out the active shaft integrated with the key, saving processing time and process, and facilitating the later physical assembly.

(3) Steering device. In the case where the position of the robot body is not offset, the rotation of the robot can be performed while the robot performs the translational

Published by Francis Academic Press, UK

-29- 
movement by connecting the two links to the rotation axis of the robot at the fixed position.

(4) The cabinet. The front and rear panels of the design box function as a fixed position and cannot be discarded. FDM desktop-level 3D printers using PLA materials cannot print transparent materials. Therefore, a transparent acrylic plate can be used on the overall structure of the cabinet to solve the ornamental problem.

\section{Simulation and processing}

\subsection{Simulation analysis}

Use the Toolbox plugin in SolidWorks to enter the design parameters of the cam in the text to generate the corresponding cam. The cam mechanism is assembled as shown in Figure 7.

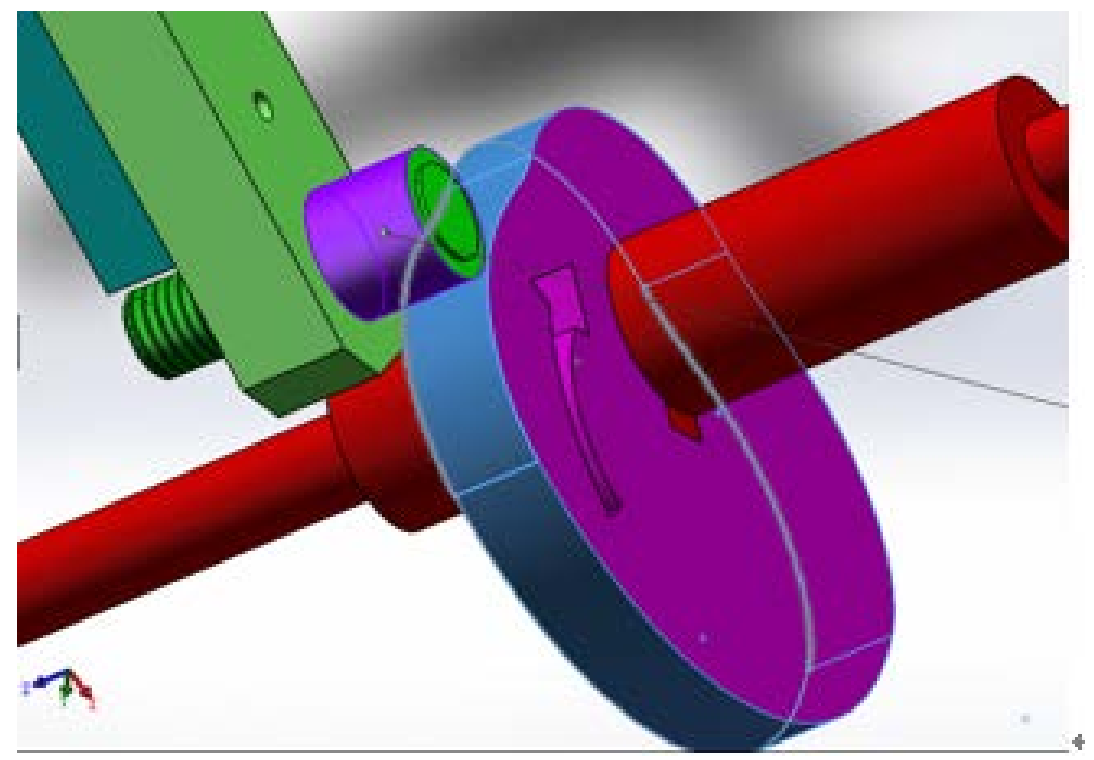

Figure. 7 Cam mechanism assembly diagram

The model is imported into the kinematics analysis software ADAMS, the rotary motor is added at the drive shaft, the whole motion simulation can be completed by calculating the motion example, and the motion trajectory as shown in Fig. 8 is obtained through post-processing, the angular velocity of the follower and The motion law of angular acceleration with time is shown in Fig. 9.

Published by Francis Academic Press, UK 


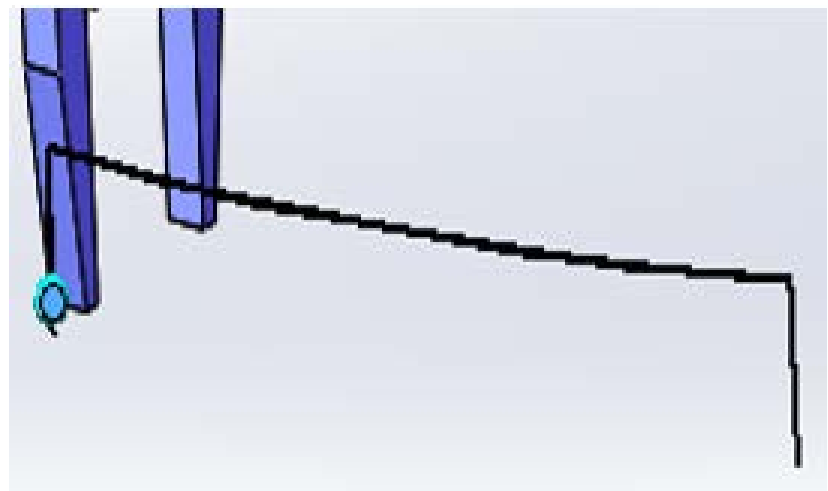

Figure. 8 Robot movement track

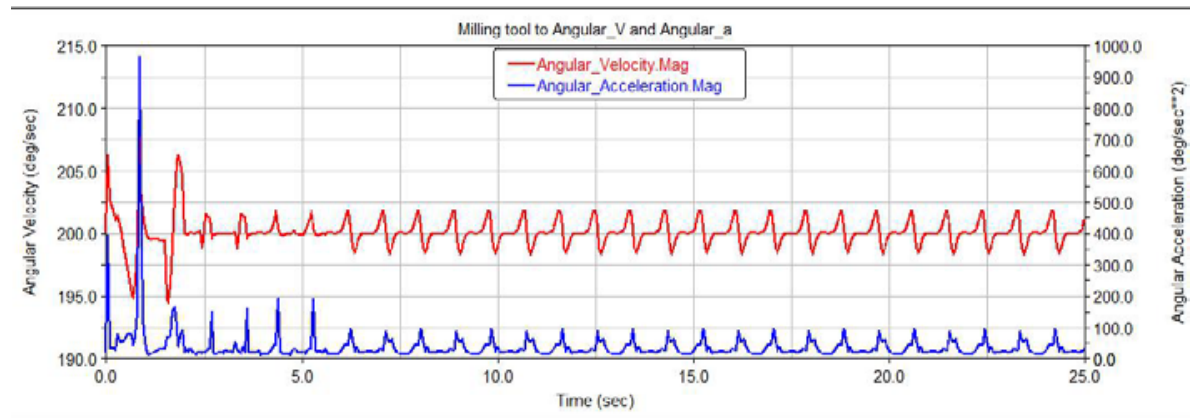

Figure. 9 Angular velocity and angular acceleration as a function of time

It can be seen from the figure that the acceleration curve of the cam follower movement is continuous, slightly abrupt sharp angle, but the peak value is small and the impact is small, indicating that the cam contour curve designed here meets the design requirements.

\subsection{Physical printing}

Use the Repetier-host software to load the saved STL file, select Slic3r to configure it, and select Generate support material in the Support material to generate support during slicing. After the slice is set, import the G code into the 3D printer to print out the entity. After surface treatment, it can be assembled and used. The printing effect is shown in Figure 10. 


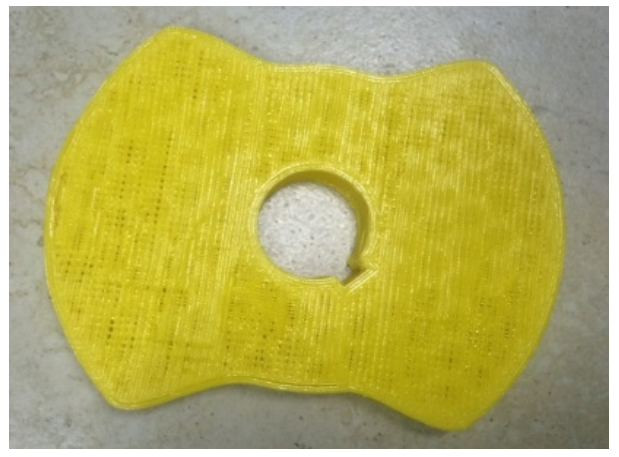

Figure. $103 D$ print cam

\section{Conclusion}

This paper uses SolidWorks to complete the design of the cam and the modeling, assembly and simulation of the cam-driven manipulator, and carried out a certain 3D printing experiment. As a demonstration teaching aid, it is easy to operate, easy to carry, suitable for students to observe and learn, and the motion track of this design can be changed by modifying the contour of the cam, and further understanding of the basic knowledge of the design of the cam profile curve and the motion law of the follower. Inspired by the process of understanding, to achieve the purpose of teaching, to help students in the conceptual design of the development of mechanical creative portfolio based on 3D printing.

\section{Acknowledgments}

Xinwen Li (1993-), male, from Suzhou, Anhui Province, majoring in robotics, ultra-high-speed magnetic suspension electric spindle technology. E-mail: 498996730@qq.com

\section{References}

[1] Zhang Xuejun. Research Status and Key Technologies of 3D Printing Technology [J]. Materials Engineering, 2016, (2): 122-128.

[2] Luo Jun. 3D printing primary textbook [M]. Infinite 3D (Qingdao) Printing Technology Industry Research Institute, 2015.6.

[3] Zhang Jing. Research on Teaching Design of Cultivating Creative Thinking by 3D Printing [D]. Shenyang Normal University. 2016.

[4] Wang Wei. Research on creative thinking of primary and middle school students [D]. Shijiazhuang: Hebei Normal University, 2013.

[5] Lin Yunfeng. Analysis of Case-based Teaching of 3D Printing in Mechanical Design [J]. Education and Teaching Forum, 2016, (29): 198-199. 
[6] Chen Yuwu. Creative mechanical structure design [J]. Nonferrous equipment, 1994, (04).

[7] Peng Zhiting. Design of demonstration teaching aids for cam mechanism in mechanical principle [J]. Science and Technology Vision, 2016, (18): 76-77.

[8] Chen Bingzhen. Discussion on creative mechanical structure design [J]. China High-tech Enterprise, 2015, (29): 32-33.

[9] Yuan Wei. New Cam Manipulator [J]. Light Industry Machinery, 2012, (6): 7578.

[10] Chen Ruiliang. Analytical Method Design of Cam-Link Combination Mechanism of Swinging Follower [J]. Mechanical Design, 2003, (29): 45-46.

[11] Ge Zhenghao. Research on Direct-acting Cam Manipulator [J]. Journal of Northwest University of Light Industry. 2002, (5): 17-20. 